\title{
Changes in the concentration of methane in the ecosystem in vitro against the background of Asteraceae family plants biomass
}

\author{
E.V. Sheida, V.A. Ryazanov, K.S. Denisenko, and O.V. Shoshina \\ Federal Research Centre of Biological Systems and Agrotechnologies, RAS, Orenburg, Russia
}

\begin{abstract}
The assessment of Asteráceae Family Plants (rhizomes and roots of elecampane and wormwood) influence on the process of methane formation in the rumen ecosystem and metabolic processes was carried out. Studies (in vitro) were carried out using ANKOM Daisy II incubator (modifications D200 and D200I) according to a specialized method. Rumen contents were obtained from beef bulls with chronic rumen fistula. Gas analysis of air and volatile fatty acids samples was performed by gas chromatography. The results of the study showed that different dosages of phytobiotic preparations did not significantly affect the characteristics of fermentation in vitro. Phytobiotic preparations of elecampane and wormwood reduce the production of methane in the ruminal fluid, which may be associated with various active components or dosages of their administration.
\end{abstract}

\section{Introduction}

The problem of global warming requires immediate resolution, and a significant role in this problem is assigned to the issues of reducing methane emissions into the atmosphere. It has been established that the livestock industry accounts for approximately $25 \%$ of the global anthropogenic methane emissions [1] and decrease in methane production by ruminants will greatly contribute to a decrease in it in the atmosphere. It will also reduce energy losses [2]. Various strategies are known to reduce the release of methane from the rumen, such as vaccination, inhibition by 2 -bromoethanesulfonic acid, antibiotics, stimulation, defaunization, adaptation to diet, use of propionate precursors or alternative electron acceptors [3].

Due to reactivity phytobiotic preparations (plant extracts, pigments, antioxidants) can play the role of electron absorbers under anaerobic conditions in the rumen and contribute to decrease in methane production [4].

Food additives that modify rumen microbial fermentation can be used to improve the growth and health of animals. According to [5], most of the 13 million $\mathrm{kg}$ of antibiotics administered to animals in 2010 worldwide were used to stimulate the growth of livestock. However, the potential for bacterial resistance has sparked intense debate over use of antibiotics, highlighting the importance of natural and safe alternatives such as probiotics, prebiotics or phytobiotics [6]. It has been shown that addition of herbal anti-MON drugs had a similar effect on rumen fermentation and growth rates in young Holstein bulls consuming 
large amounts of concentrate [7]. Another study [8] showed a similar effect of neomycin and origanum leaves on the number of days of brushing, the severity of brushing, and mortality in newborn calves. Plants or their biologically active compounds with antimicrobial properties can improve feed utilization and animal performance by altering rumen microbial fermentation (for example, short-chain fatty acid profile, methane production, nitrogen metabolism or $\mathrm{pH}[9]$.

Herbs exhibit not only antibacterial effects that modulate rumen fermentation, but a wide range of health benefits such as antioxidant and anti-inflammatory effects [10]. In addition, plant products are currently used in the feed industry mainly as sensory additives, flavorings, and appetizing substances [11].

Purpose of the study: in our study, we looked for new natural substances to reduce production of methane in the rumen and assessed whether the selected plant materials affect metabolic processes in the rumen and methanogenesis.

\section{Materials and Methods}

The object of the study is the ruminal contents (RC) obtained from bulls of the Kazakh white-headed breed with a chronic fistula of the rumen, with an average weight of 220$225 \mathrm{~kg}$, at the age of 9-10 months.

Maintenance of animals and experimental studies were carried out in accordance with the instructions and recommendations of Russian regulations (1987; Order of the Ministry of Health of the USSR No. 755 dated August 12, 1977 "On Measures to Further Improve Organizational Forms of Work using Experimental Animals") and Guide for the Carre and Use of Laboratjry Animals (National Academy Press, Washington, D.C., 1996). Within the studies, measures were taken to minimize the suffering of animals and reduce the number of tested samples.

For the study, we used Inulae rhizomata et radices (elecampane of rhizomes and roots), Artemisiae absinthil herba (wormwood, bitter herb).

Experiment Scheme. The studies were carried out by the in vitro method using the device - ANKOM Daisy II incubator (modifications D200 and D200I) according to the specialized method. Distilled water was chosen as the dispersion medium. Each experiment was carried out in three repetitions. After incubation, air samples were taken to determine the level of methane on Kristallux-2000M device by gas chromatography.

Laboratory studies were carried out at the Testing Center of the Center for Collective Use of the Federal Research Center of the BST of the RAS: the level of volatile fatty acids (VFA) in the contents of the rumen was determined by gas chromatography using gas chromatograph Crystallux-4000M, determination of nitrogen forms - according to GOST 26180-84.

Statistical processing. The numerical data were processed using the SPSS Statistics 20 software (IBM, USA), the mean (M), standard deviations $( \pm \sigma)$, and standard deviation errors $( \pm \mathrm{SE})$ were calculated. To compare the options, a nonparametric method of analysis was used. Differences were considered statistically significant at $\mathrm{p} \leq 0.05$.

\section{Study Results}

Within in vitro studies, it was found that the VFA level in the samples with introduction of various dosages of phytobiotic preparations was different (Table 1). The rhizomes and roots of elecampane in all studied dosages and wormwood herb at a dose of $2.0 \mathrm{~g}$ and $5.0 \mathrm{~g}$ showed the same level of acetic acid in the ruminal fluid. The concentration of acetic acid in the sample with the presence of wormwood herb at a dosage of $10 \mathrm{~g} / \mathrm{kg} \mathrm{DM}$ was significantly 
higher than in other samples by $79.2 \%(\mathrm{p} \leq 0.05)$; it shall also be noted that the concentration of all detected VFAs in this group was higher than other samples. The same level of propionic acid was recorded in samples 2, 3, 4 and 5, its concentration was higher by $56.7 \%$ in sample 1 and by $18.8 \%$ in sample 6 . A low concentration of butyric acid was shown in samples containing elecampane $6.0 \mathrm{~g}$ and wormwood $5.0 \mathrm{~g}$ and $2.0 \mathrm{~g}$, in the presence of wormwood at a dose of $10.0 \mathrm{~g}$, the level of this acid increased by $90.5 \%(\mathrm{p} \leq 0.01)$.

The VFA concentration in the ruminal fluid was noted $64.6 \%$ lower relative to sample 6 in the study of sample 1 . The lowest VFA level was recorded in a sample with wormwood grass at a dosage of $5.0 \mathrm{~g}$, the level of valeric and caproic acids in this group was minimal and amounted to 0.9 and $0.8 \mathrm{mmol} / 100 \mathrm{ml}$.

Table 1. Concentration of volatile fatty acids in ruminal fluid, $\mathrm{mmol} / 100 \mathrm{ml}$

\begin{tabular}{|c|c|c|c|c|c|c|c|}
\hline \multirow{2}{*}{$\begin{array}{l}\dot{0} \\
0 \\
\stackrel{0}{0} \\
\text { : }\end{array}$} & \multirow[t]{2}{*}{ Name } & \multirow{2}{*}{$\begin{array}{l}\text { Dose, } \\
\mathrm{g} / \mathrm{kg} \\
\text { DM }\end{array}$} & \multicolumn{5}{|c|}{ Volatile fatty acids, $\mathrm{mmol} / 100 \mathrm{ml}$} \\
\hline & & & Acetic & Propionic & Butyric & Valeric & Caproic \\
\hline 1 & \multirow{3}{*}{$\begin{array}{l}\text { Inulae } \\
\text { rhizomat } \\
a \quad \text { et } \\
\text { radices }\end{array}$} & 3.0 & $1.6 \pm 0.002$ & $3.0 \pm 0.001$ & $2.9 \pm 0.004$ & $2.1 \pm 0.006$ & $1.1 \pm 0.0002$ \\
\hline 2 & & 1.0 & $1.6 \pm 0.004$ & $1.3 \pm 0.002$ & $2.6 \pm 0.006$ & $1.2 \pm 0.0004$ & $0.8 \pm 0.0002^{* *}$ \\
\hline 3 & & 6.0 & $1.6 \pm 0.004$ & $1.3 \pm 0.001$ & $1.1 \pm 0.001^{*}$ & $0.9 \pm 0.0004$ & $2.9 \pm 0.006^{*}$ \\
\hline 4 & \multirow{3}{*}{$\begin{array}{l}\text { Artemisi } \\
\text { ae } \\
\text { absinthil } \\
\text { herba }\end{array}$} & 5.0 & $1.6 \pm 0.012$ & $1.3 \pm 0.002$ & $1.1 \pm 0.001^{*}$ & $0.9 \pm 0.0002$ & $0.8 \pm 0.0001^{* * *}$ \\
\hline 5 & & 2.0 & $1.6 \pm 0.006$ & $1.3 \pm 0.001$ & $1.1 \pm 0.002 *$ & $1.4 \pm 0.002$ & $4.0 \pm 0.004 *$ \\
\hline 6 & & 10.0 & $7.7 \pm 0.004 *$ & $1.6 \pm 0.004$ & $\begin{array}{l}11.6 \pm 0.004 \\
* *\end{array}$ & $6.3 \pm 0.002$ & $3.0 \pm 0.002 *$ \\
\hline
\end{tabular}

The content of nitrogenous components of the ruminal fluid (RF) is one of the indicators of the degree of assimilation of nitrogen in the feed, as well as the general direction of the ruminal digestion processes. The highest concentration of total nitrogen in the ruminal fluid was noted in sample 4, and the lowest one in samples 1 and 2. The wormwood herb showed a higher content of total, non-protein and ammoniacal nitrogen in the ruminal fluid than the rhizomes and roots of elecampane, and regardless of the concentration (Figure 1). A direct relationship was found between the concentration of total and protein nitrogen. Protein nitrogen was approximately at the same level in samples $1,3,5$ and 6 , in sample 4 it increased by $45.1 \%-47.7 \%$, in sample 2 - by $15.7 \%-19.7 \%$.

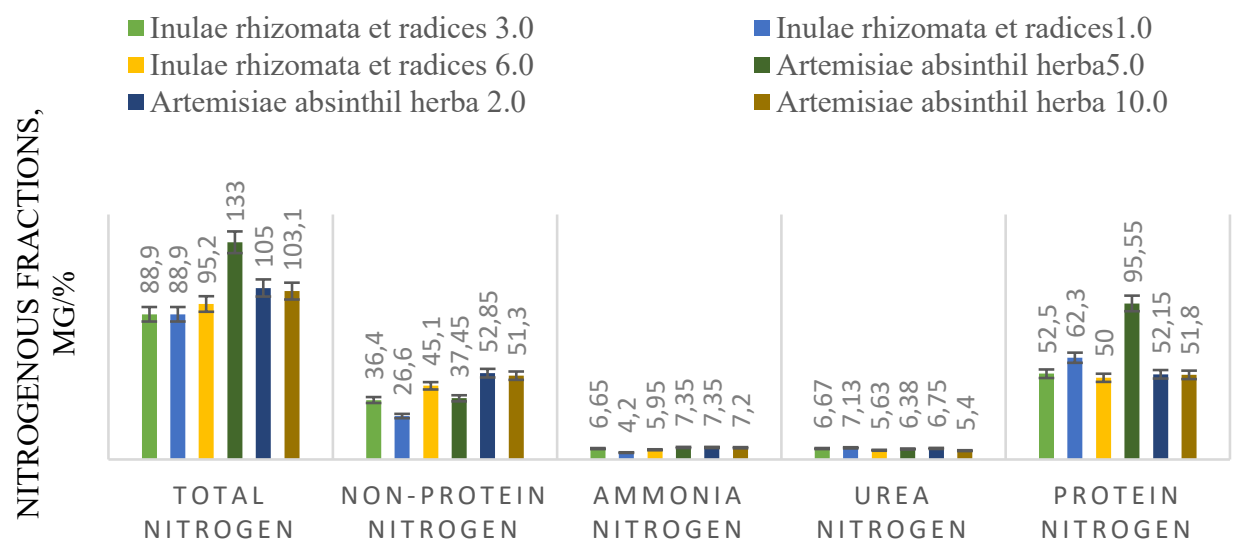

Fig. 1. Content of nitrogenous fractions in ruminal fluid, $\mathrm{mg} / \%$. 
Wormwood herb at a dosage of $10.0 \mathrm{~g}$ per $1 \mathrm{~kg}$ of DM reduced methane production to a greater extent than its other dosages $(\mathrm{p} \leq 0.05)$, so a dose of $2.0 \mathrm{~g}$ showed an increase in the methane level in the ruminal fluid by $36.5 \%$ ( $\leq 0.01)$, and the dose of $5.0 \mathrm{~g}-47.5 \%$ $(\mathrm{p} \leq 0.01)$. Rhizomes and roots of elecampane in various dosages showed a high level of methane production, however, the min and max doses of this phytobiotic showed a $38.1 \%$ $38.6 \%$ decrease in methane in the ruminal fluid (Table 2). In all groups, there was a direct relationship between the level of $\mathrm{CH}_{4}$ and $\mathrm{CO}_{2}$.

Table 2. Concentration of methane $\left(\mathrm{CH}_{4}\right), \mathrm{mg} / \mathrm{m}^{3}$ and $\mathrm{CO}_{2}$, e/g under in vitro conditions

\begin{tabular}{|c|c|c|c|}
\hline \multirow{2}{*}{ Title } & Dose, $\mathrm{g} / 1 \mathrm{~kg}$ of & \multicolumn{2}{|c|}{ Concentration $\mathrm{CH}_{4}$ in vitro } \\
\cline { 2 - 4 } & $\mathrm{DM}$ & $\mathrm{CH}_{4}, \mathrm{mg} / \mathrm{m}^{3}$ & Equivalent $\mathrm{CO}_{2}$ e/g \\
\hline \multirow{3}{*}{ Inulae rhizomata et radices } & 3,0 & $21500 \pm 10,26$ & $537,5 \pm 4,36$ \\
\cline { 2 - 4 } & 1,0 & $13300 \pm 9,74^{*}$ & $332,5 \pm 5,12^{*}$ \\
\cline { 2 - 4 } & 6,0 & $13200 \pm 9,16^{*}$ & $330 \pm 4,22^{*}$ \\
\hline \multirow{2}{*}{ Artemisiae absinthil herba } & 5,0 & $17900 \pm 10,54^{*}$ & $447,5 \pm 5,16^{*}$ \\
\cline { 2 - 4 } & 2,0 & $14800 \pm 10,12^{*}$ & $370 \pm 4,26^{*}$ \\
\cline { 2 - 4 } & 10,0 & $9400 \pm 8,58^{*}$ & $235 \pm 4,10^{*}$ \\
\hline
\end{tabular}

\section{Discussion}

Herbal preparations or their active ingredients are characterized by antimicrobial activity against both gram-negative and gram-positive bacteria, as well as antifungal activity [12]. We assume that in our experiment, the probable mechanism of action is inhibition of deamination and methanogenesis, leading to decrease in the concentration of methane and acetate, and increase in the concentration of propionate and butyrate [10] (as a result of the addition of elecampane at a dose of $3.0 \mathrm{~g} / \mathrm{kg} \mathrm{DM}$ and wormwood in dose of $10.0 \mathrm{~g} / \mathrm{kg} \mathrm{DM}$ ). Our findings are in part consistent with those reported in other sources [13, 14]. It has been shown that low doses of biologically active plant compounds increase the concentration of butyric acid [6]; herein, this was observed at low doses of Inulae rhizomata et radices.

In in vitro study, the effect of phytobiotics and their components on the VFA profile was observed, but it was noted that they did not affect the nitrogen concentration in the ruminal fluid $[14,15]$. These authors suggested that herbal concentrations shall be higher than $35 \mathrm{mg} / \mathrm{l}$ in order to induce any significant changes in rumen nitrogen metabolism. In our study, we noted that the wormwood herb in all studied dosages contributed to increase in all nitrogenous fractions in the ruminal fluid, a similar level of nitrogen was recorded with introduction of the rhizome and roots of elecampane at the maximum dosage.

Previous studies have shown that saponins inhibit protozoa and also limit the availability of hydrogen for methanogenesis [16]. In a recent study by Holtshausen et al. [17] stated that they revealed a decrease in methane content at higher levels of inclusion of herbal preparations containing saponin (15 g/ kg DM and more); in our experiment, we showed decrease in methane at low and high doses of roots and rhizomes of elecampane (1.0 and $6.0 \mathrm{~g} / \mathrm{kg}$ DM of the diet).

Essential oils that make up phytobiotic preparations can also reduce the amount of available hydrogen for methanogenesis [18]; in the studies carried out, decrease in the level of methane was also noted with the additional inclusion of high doses $(10.0 \mathrm{~g} / \mathrm{kg} \mathrm{DM})$ of wormwood herb, which has essential oils in its composition.

\section{Conclusion}

Therefore, different dosages of dry plant biomass Inulae rhizomata et radices and Artemisiae absinthil herba did not have a significant effect on the fermentation characteristics in the 
rumen ecosystem, but they contribute to decrease in methane production in the ruminal fluid. Further study is necessary using the methods of metagenomic analysis of the rumen microbiome.

\section{Acknowledgments}

This study was performed with the financial support from the Russian Science Foundation (Project No.21-76-10014)

\section{References}

1. K.R. Lassey, Aust J Exp Agr, 48, 114-118 (2008)

2. K.A. Johnson, D.E. Johnson, J Anim Sci, 73, 2483-2492 (1995)

3. R. Bodas, S. López, M. Fernández, R. García-González, A.B. Rodríguez, R.J. Wallace, J.S. González, Anim Feed Sci Technol, 145, 245-258 (2008)

4. I. Karimov, K. Kondrashova, G. Duskaev, O. Kvan, Evaluation of effects of rumen fluid in combination with probiotic preparations and vanillin on the luminescence of a recombinant strain E. coli., E3S Web of Conferences, 143, 02034 (2020)

5. M. K. Chattopadhyay, Frontiers in Microbiology, 5, 1-3 (2014)

6. R. Khiaosa-ard, Q. Zebeli, Journal of Animal Science, 91, 1819-1830 (2013)

7. M. Devant, A. Anglada, A. Bach, Animal Feed Science and Technology, 137, 46-57 (2007)

8. V. A. Bampidis, V. Christodoulou, P. Florou-Paneri, E. Christaki, Journal of Veterinary Medicine Series A, 53, 154-156 (2006)

9. M. Akbarian-Tefaghi, E. Ghasemi, M. Khorvash, J Anim Physiol Anim Nutr (Berl). Jun, 102(3), 630-638 (2018)

10. S. Calsamiglia, M. Busquet, P. W. Cardozo, L. Castillejos, A. Ferret, Journal of Dairy Science, 90, 2580-2595 (2007)

11. C. K. Franz, H. C. Baser, W. Windisch, Flavour and Fragrance Journal, 25, 327-340 (2010)

12. K.S. Inchagova, G.K. Duskaev, D.G. Deryabin, Microbiology (Russian Federation) 88(1), 63-71 (2019)

13. D. Petrič, D. Mravčáková, K. Kucková, S. Kišidayová, A. Cieslak, M. SzumacherStrabel, H. Huang, P. Kolodziejski, A. Lukomska, S. Slusarczyk, K. Čobanová, Z. Váradyová, Front Vet Sci., 8, 630971(2021)

14. M. E. Abolfathi, S. A. Tabeidian, A. D. Foroozandeh Shahraki, S. N. Tabatabaei, M. Habibian, Arch Anim Nutr., 73(2), 88-110 (2019)

15. R. Temmar, M. Rodríguez-Prado, G. Forgeard, C. Rougier, S. Calsamiglia, Animals (Basel), 11(5), 1205 (2021)

16. F. Zhang, B. Li, Z. Ban, H. Liang, L. Li, W. Zhao, X. Yan, J Anim Physiol Anim Nutr (Berl), 105(4), 630-638 (2021)

17. K.A. Beauchemin, E.M. Ungerfeld, R.J. Eckard, M. Wang, Animal. Mar, 14(S1), 2-16 (2020)

18. K. A. Beauchemin, S. M. McGinn, Journal of Animal Science, 84(6), 1489-1496 (2006) 